\title{
A novel technique for recombinant protein expression in duckweed (Spirodela polyrhiza) turions
}

\author{
Salil Chanroj · Aompilin Jaiprasert - Nipatha Issaro
}

Received: 13 July 2021 / Revised: 17 August 2021 / Accepted: 17 August 2021

(C) Korean Society for Plant Biotechnology

\begin{abstract}
Spirodela polyrhiza, from the Lemnaceae family, are small aquatic plants that offer an alternative plant-based system for the expression of recombinant proteins. However, no turion transformation protocol has been established in this species. In this study, we exploited a pB7YWG2 vector harboring the eYFP gene that encodes enhanced yellow fluorescent protein (eYFP), which has been extensively used as a reporter and marker to visualize recombinant protein localization in plants. We adopted Agrobacterium tumefaciensmediated turion transformation via vacuum infiltration to deliver the $e Y F P$ gene to turions, special vegetative forms produced by duckweeds to endure harsh conditions. Transgenic turions regenerated several duckweed fronds that exhibited yellow fluorescent emissions under a fluorescence microscope. Western blotting verified the expression of the eYFP protein. To the best of our knowledge, this is the first report of an efficient protocol for generating transgenic $S$. polyrhiza expressing eYFP via Agrobacterium tumefaciensmediated turion transformation. The ability of turions to withstand harsh conditions increases the portability and versatility of transgenic duckweeds, favoring their use in the further development of therapeutic compounds in plants.
\end{abstract}

Keywords Agrobacterium tumefaciens-mediated turion transformation, Enhanced yellow fluorescent protein, Fluorescence microscopy, Spirodela polyrhiza, Turion, Vacuum infiltration

S. Chanroj

Department of Biotechnology, Faculty of Sciences, Burapha

University, Chonburi, 20131 Thailand

A. Jaiprasert

Department of Biotechnology, Faculty of Sciences, Burapha

University, Chonburi, 20131 Thailand

N. Issaro $(\bowtie)$

Division of Pharmacognosy and Pharmaceutical Chemistry,

Faculty of Pharmaceutical Sciences, Burapha University,

Chonburi, 20131 Thailand

e-mail: nipatha@go.buu.ac.th

\section{Introduction}

Duckweeds, members of the family Lemnaceae, are small aquatic free-floating plants that are widely distributed on the surface of slow-flowing water (Tang et al. 2014). They are the smallest angiosperms and consist of 38 species in 5 genera (Appenroth et al. 2013; Wang 2016), namely, Lemna, Landoltia, Spirodela, Wolffiella, and Wolffia. Members of the genera Spirodela, Landoltia, and Lemna have only fronds and one to a few roots, whereas the genera Wolffiella and Wolffia have no roots (Landolt 1986). The leaf-like structures of duckweeds termed fronds are small (Wang et al. 2015), but the mature fronds of Spirodela polyrhiza ( $S$. polyrhiza), the largest among the duckweed species, average 4- to 10-mm long (Oláh et al. 2008). The fronds of $S$. polyrhiza reproduce asexually through budding of offspring (daughter) fronds from the meristematic pocket to produce true turions (Gordon-Kamm et al. 1990; Lemon et al. 2000; Mejbel and Simon 2018).

The buds form true turions during winters in the dominant phase (Hillman 1961; Krajnčič and Slekovec-Golob 1991; Krajnčič and Devidé 1979; Wang and Messing 2012; Wang et al. 2014). These turions play a significant role in the survival of vegetative fronds by sinking to the bottom of the water and germinating new fronds under suitable conditions (Landolt 1986; Landolt and Kandcler 1987).

Recently, S. polyrhiza has been utilized as an efficient plant expression system for the production of protein antigens in the area of biopharmaceutical plant research (Thu et al. 2015) as it is fast-growing, has a short life span (Wang et al. 2014; Lemon et al. 2001), and has no genetic variation (Yang et al. 2021). Therefore, efficient $\beta$-glucuronidase (GUS) gene transformation protocols have been developed for S. polyrhiza in callus using an Agrobacterium tumefaciens-mediated callus transformation method (Yang and $\mathrm{Li}$ et al. 2018). However, there have been no techniques published for the Agrobacterium tumefaciens-mediated trans- 
formation of turions of $S$. polyrhiza.

The gene coding for green fluorescent protein (GFP) from the jellyfish Aequorea victoria has been successfully used as a marker for duckweed transformation (Cantó-Pastor et al. 2015; Vunsh et al. 2007).

GFP transgene expression was stable over multiple subcultures for plant species, as the GUS system is unsuitable for the rapid screening of primary transformants of living plants (Jefferson et al. 1987). Yellow fluorescent protein (YFP) is a variant of GFP fabricated by changing some amino acid residues, shifting the protein to produce a yellowish emission (Ormö et al. 1996; Tsien 1998). Enhanced yellow florescent protein (eYFP) is a modified YFP with an increased quantum yield. It is currently the most widely used version of fluorescent protein (Jusuk et al. 2015). Generally, eYFP gene and genes conferring resistance to antibiotics or herbicides are introduced to plant cells using A. tumefaciens-mediated transformation due to the simplicity, reliability, and effectiveness of this technique. Based on herbicides, resistant genes are commonly used to transform selected transgenic plants (Áy et al. 2021). Such as the bialaphos-resistance ( $b a r$ ) gene was used to construct transgenic plants resistant to herbicide glufosinate, which is the active ingredient of the commercial herbicide BASTA ${ }^{\circledR}$ (Dröge et al. 1992), and many researchers have heavily used it for genetic modification of various plant crops (Christou et al. 1991; Gordon-Kamm et al. 1990; Janakiraman et al. 2002; Tan et al. 2006; Vasil et al. 1992). The examples of $A$. tumefaciens-mediated transformation protocols have been reported for S. polyrhiza callus (Yang and Li et al. 2018) and fronds (Thu et al. 2015) to produce transgenic $S$. polyrhiza which is resistant to antibiotics. However, $A$. tumefaciens-mediated turion transformation in this species has been poorly characterized and transgenic $S$. polyrhiza resistant to the herbicide glufosinate has not been widely reported so far.

In our previously report, we optimized many factors important for the GFP transformation of $S$. polyrhiza turions, such as the preculture period, the turion induction conditions, the culture media, Agrobacterium density, the effect of ceftriaxone on Agrobacterium growth, acetosyringon concentration, and herbicide glufosinate concentration, to regenerate transgenic duckweeds (Jaiprasert 2008). The efficiency of GFP transformation of Agrobacteria was improved by up to $75 \%$ using vacuum infiltration, but the protein expression of $S$. polyrhiza turions was not confirmed. In this study, we aimed to create transgenic $S$. polyrhiza expressing recombinant eYFP through $A$. tumefaciens-mediated turion transformation resistant to herbicide glufosinate using $16 \mathrm{~h}$ light $/ 8 \mathrm{~h}$ dark and dark conditions during co-cultivation. Protein expression was determined using SDS-PAGE and western blot analysis. These techniques should be portable and capable of tolerating harsh conditions, paving the way for the development of mobile pharmaceutical plant factories in the near future.

\section{Materials and Methods}

Plant materials and cultivation of S. polyrhiza

S. polyrhiza were collected from natural reservoirs in Burapha University, Chonburi, Thailand. They were sterilized in $0.9 \%$ sodium hypochlorite $(\mathrm{NaOCl})$ with $0.05 \%$ Tween 20 for 2 minutes and washed five times with sterile deionized water. After washing, 10 fronds of $S$. polyrhiza were cultured on solid Murashige-Skoog (MS) medium (PhytoTech, Lenexa, $\mathrm{KS}$, USA) and incubated at $25^{\circ} \mathrm{C} \pm 2{ }^{\circ} \mathrm{C}$, with a $16 \mathrm{~h} \mathrm{light} / 8 \mathrm{~h}$ dark photoperiod with $55 \mu \mathrm{mol} \mathrm{m} \mathrm{m}^{-2} \mathrm{~s}^{-1}$ from fluorescent light bulbs. After 14 days, S. polyrhiza was transferred to Hoagland's E medium and cultivated for 14 days (Jaiprasert 2008).

Turion induction

S. polyrhiza was cultured under starvation conditions to induce turion formation. Turions are morphologically different from fronds but can regenerate to form fronds when the required nutrients are replenished. In this experiment, 15-16 fronds of $S$. polyrhiza were left to starve in sterile deionized water under $16 \mathrm{~h}$ light $/ 8 \mathrm{~h}$ dark photoperiods with an irradiation of $55 \mu \mathrm{mol} \mathrm{m} \mathrm{m}^{-2} \mathrm{~s}^{-1}$ from fluorescent light bulbs at $25^{\circ} \mathrm{C} \pm 2^{\circ} \mathrm{C}$. Turions started to form within 7 days of starvation and sunk to the bottom of the bottles within 45 days (Jaiprasert 2008).

\section{Verification of plasmid DNA using PCR}

eYFP was amplified using polymerase chain reaction (PCR)specific primers. The eYFP forward primer was 5'-ACCA TGTGATCGCGCT- $3^{\prime}$, and the eYFP reverse primer was 5'-TGAACCGCATCGAGC-3'. PCR was performed in a MultiGene OptiMax thermal cycler (Labnet International, Inc., NJ, UK) as follows: the temperature was $94^{\circ} \mathrm{C}$ during denaturation for $2 \mathrm{~min}, 40$ cycles of $94^{\circ} \mathrm{C}$ during denaturation for $30 \mathrm{~s}, 72^{\circ} \mathrm{C}$ during denaturation for $30 \mathrm{~s}$, annealing temperature of $51^{\circ} \mathrm{C}$ for $30 \mathrm{~s}$, and final extension time at $72^{\circ} \mathrm{C}$ for 7 min (Jaiprasert 2008). The PCR product was mixed with 6X DNA loading dye and separated on $1 \%$ agarose gels. Finally, the DNA was stained with SYBR ${ }^{\mathrm{TM}}$ 
Safe DNA Gel Stain (Invitrogen, Carlsbad, CA, USA) and visualized on a BLooK LED transilluminator at $470 \mathrm{~nm}$ (GeneDireX, Inc., Taoyuan, Taiwan).

Agrobacterium strain and vector

The binary vector pB7YWG2 (Fig. 1) (Kirami et al. 2002) was used to transform A. tumefaciens strain GV3101 and to deliver the $e Y F P$ gene to $S$. polyrhiza for turion transformation. The expression vector pB7YWG2 consisted of the CaMV 35S promoter to drive the expression of the eYFP gene, a spectinomycin resistance $\left(\mathrm{Sp}^{\mathrm{R}}\right)$ gene for Escherichia coli selection and a bialaphos-resistance (bar) gene for plant selection. A single colony of pB7YWG2 vector after manipulation in $E$. coli $\mathrm{DH} 5 \alpha$ was confirmed using an $e Y F P$ gene-specific primer to confirm the presence of the pB7YWG2 plasmid.

Agrobacterium transformation

Agrobacterium stock was grown on Luria-Bertani (LB) agar medium supplemented with $50 \mu \mathrm{g} / \mathrm{mL}$ of gentamicin and incubated at $30^{\circ} \mathrm{C}$ for 2 days. The corrected plasmid pB7YWG2 was transferred into A. tumefaciens strain GV3101 via heat-shock transformation as described by Sambrook and colleagues (Sambrook et al. 1989), with slight modifications. Briefly, $1.0 \mu \mathrm{g}$ of plasmid pB7YWG2 was added to $50 \mu \mathrm{L}$ of Agrobacterium-competent cells, and the mixture was incubated at $42^{\circ} \mathrm{C}$ for $2 \mathrm{~min}$ and then placed on ice for $30 \mathrm{~min}$. The transformants were spread on LB agar supplemented with $50 \mu \mathrm{g} / \mathrm{mL}$ of gentamycin and $200 \mu \mathrm{g} / \mathrm{mL}$ of spectinomycin and then incubated at $30^{\circ} \mathrm{C} \pm 2^{\circ} \mathrm{C}$ for 2 days. The white colonies of single transformed Agrobacterium carrying plasmid pB7YWG2 were then inoculated into LB liquid medium supplemented with the concentration of antibiotics indicated above. The transformants were incubated at $30^{\circ} \mathrm{C} \pm 2^{\circ} \mathrm{C}$ overnight with shaking at $200 \mathrm{rpm}$, subcultured at $10 \%$ inoculum, and cultivated until the $\mathrm{OD}_{600}$ reached 2.0 (Jaiprasert 2008).

\section{Turion transformation}

The cell pellet of Agrobacterium carrying plasmid pB7YWG2 was harvested via centrifugation at 5,000 rpm and resuspended in MS medium supplemented with $1 \%$ sucrose, $100 \mu \mathrm{M}$ acetosyringone (Sigma-Aldrich, St. Louis, MO, USA), and $0.2 \%$ Tween 80 . The cell density was adjusted to 0.5 at an optical density of $600 \mathrm{~nm}$ (Jaiprasert 2008). The turions were immersed in Agrobacterium suspension and vacuum-infiltrated at $-60 \mathrm{mmHg}$ for $10 \mathrm{~min}$ (Yang et al. 2018; Yang and Li et al. 2018) before being incubated at room temperature for $30 \mathrm{~min}$. The infiltrated turions were blotted dry on sterile filter paper and co-cultivated on filter paper discs soaked with $10-\mathrm{mL}$ MS liquid medium supplemented with $1 \%$ sucrose and $100-\mu \mathrm{M}$ acetosyringone (Sigma-Aldrich) (Jaiprasert 2008) and then incubated under either $16 \mathrm{~h}$ light/ $8 \mathrm{~h}$ dark or dark conditions for 3 days (Chhabra et al. 2011; Thu et al. 2015; Yang et al. 2018). The co-cultivated turions were gently washed four to five times with sterile deionized water containing $750 \mathrm{mg} / \mathrm{L}$
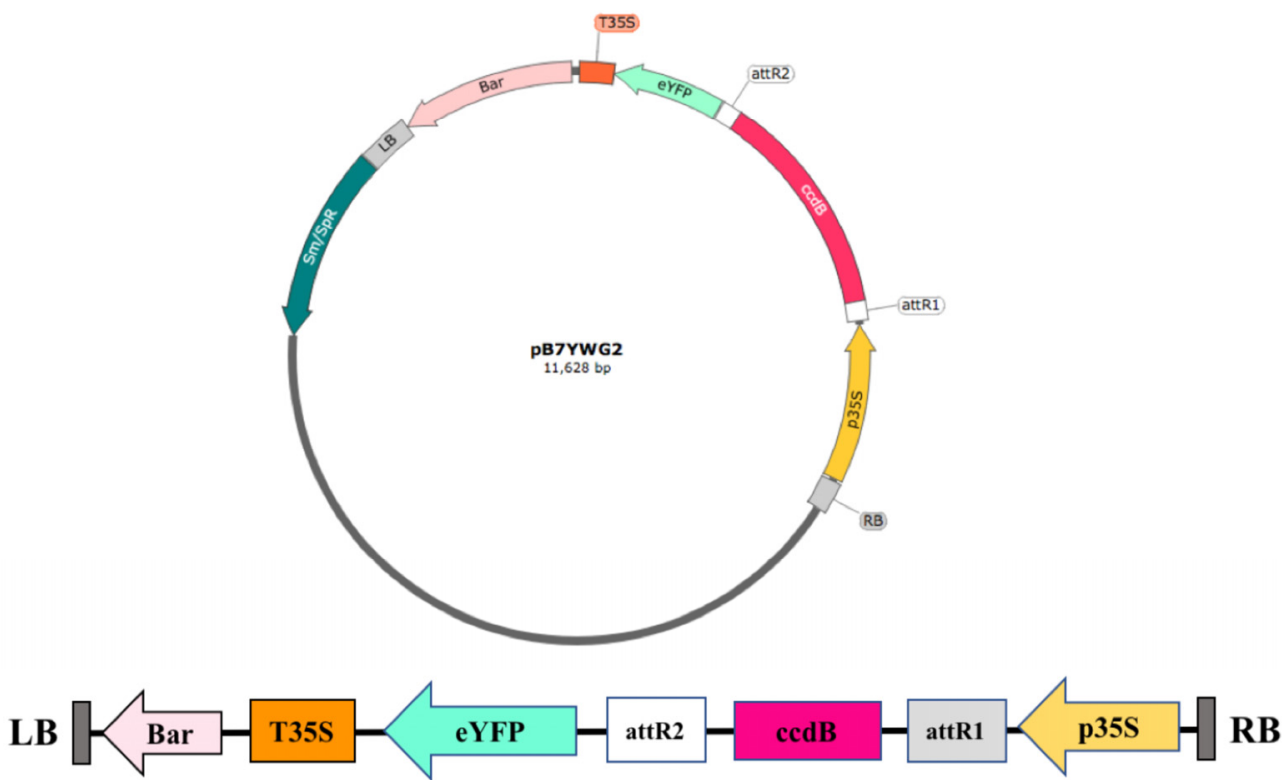

Fig. 1 The pB7YWG2 mapping we used for turion transformation in S. polyrhiza 
ceftriaxone to remove excess Agrobacterium and dried on sterile filter paper. Ten transformed turions were then transferred to selective MS agar media; MS2 (MS, 1\% sucrose, $100-\mu \mathrm{M}$ acetosyringone (Sigma-Aldrich), 250-mg/L ceftriaxone, $0.8 \%$ agar, $\mathrm{pH} 5.8$ ) or MS3 (MS, $1 \%$ sucrose, $100-\mu \mathrm{M}$ acetosyringone (Sigma-Aldrich), 250-mg/L ceftriaxone, 0.01-mM glufosinate, $0.8 \%$ agar, $\mathrm{pH} 5.8$ ). The negative controls of wild-type turions were cultured on MS1 agar medium (MS, $1 \%$ sucrose, $0.8 \%$ agar, $\mathrm{pH}$ 5.8) (Jaiprasert 2008). All turions were cultured under a $16 \mathrm{~h}$ light $/ 8 \mathrm{~h}$ photoperiod with an irradiation of $55 \mu \mathrm{mol} \mathrm{m} \mathrm{m}^{-2} \mathrm{~s}^{-1}$ from fluorescent light bulbs at $25^{\circ} \mathrm{C} \pm 2{ }^{\circ} \mathrm{C}$ for 14 days.

eYFP detection under fluorescence microscope

Transgenic $S$. polyrhiza freshly regenerated from transformed turions were mounted on slides filled with deionized water. Yellow fluorescence and chlorophyll autofluorescence were detected using an excitation filter BP450-480 (blue) and a barrier filter BA515 (green and red) under a fluorescence microscope (Olympus BX51, Shinjuku City, Tokyo, Japan). Chlorophyll autofluorescence was detected using an excitation filter BP510-550 (green) and a barrier filter BA590 (red) for control purposes. Images were captured using an Olympus DP22 digital camera.

Protein extraction and western blotting analysis

Regenerated fronds of 100-mg transgenic S. polyrhiza were ground in $200-\mu \mathrm{L}$ lysis buffer containing $50-\mathrm{mM}$ Tris, $300-\mathrm{mM} \mathrm{NaCl}$, and $2 \%$ glycerol at $\mathrm{pH} 8.0$ for $30 \mathrm{~min}$ via homogenization on ice. The supernatant was collected after centrifugation at $15,000 \mathrm{rpm}$ at $4^{\circ} \mathrm{C}$ for $20 \mathrm{~min}$ and kept at $-20^{\circ} \mathrm{C}$. This supernatant of the protein extraction was used for western blotting. Proteins were separated on $12 \%$ SDS-PAGE gels and transferred to PVDF membranes (BioRad Hercules, CA, USA). After blocking with 5\% non-fat dried milk, the blots were incubated with rabbit anti-green fluorescent protein (GFP) antibody in 1:1000 dilution (Cell Signaling Technology, Danvers, MA, USA), followed by incubation with goat anti-rabbit immunoglobulin $\mathrm{G}$ coupled to horseradish peroxidase in 1:1000 dilution (Cell Signaling Technology). Immunoreactive signals were detected via chemiluminescence (Santa Cruz Biotechnology, Dallas, TX, USA) according to the manufacturer's protocols.

Statistical analysis

The transformation efficiency was calculated as the number of regenerated fronds of transgenic $S$. polyrhiza on selective media/number of all turions inoculated with Agrobacterium $\times 100(\%)$. The transformation efficiency data were evaluated using one-way ANOVA, followed by a Tukey's multiple comparisons test. A $P$ value of $<0.05$ was considered to indicate statistical significance. All visualization and statistical analyses were performed using GraphPad Prism software version 5.0 for Windows (GraphPad Software, San Diego, California, USA).

\section{Results}

S. polyrhiza cultivation and turion induction

To cultivate duckweeds axenically in the laboratory, the young fronds of S. polyrhiza were submerged in $0.90 \%$ $\mathrm{NaClO}$ for $2 \mathrm{~min}$ (Fig. 2A). $\mathrm{NaClO}$ solution was utilized as a sterilizing agent for the surface sterilization of $S$. polyrhiza. Turions are special organs of some species of duckweeds developed for survival under a stressful condition. To induce turion formation, fronds of $S$. polyrhiza were subjected to starvation in sterile deionized water for 45 days. After 7 days, the fronds began to undergo chlorosis, developing a yellow color, with reddish pigments accumulating on the ventral (abaxial) side. After 45 days, turions were observed as dark-green colored organs attached to the mother fronds (Fig. 2B). Subsequently, the turions sunk to the bottom of the vessels (Fig. 2C).

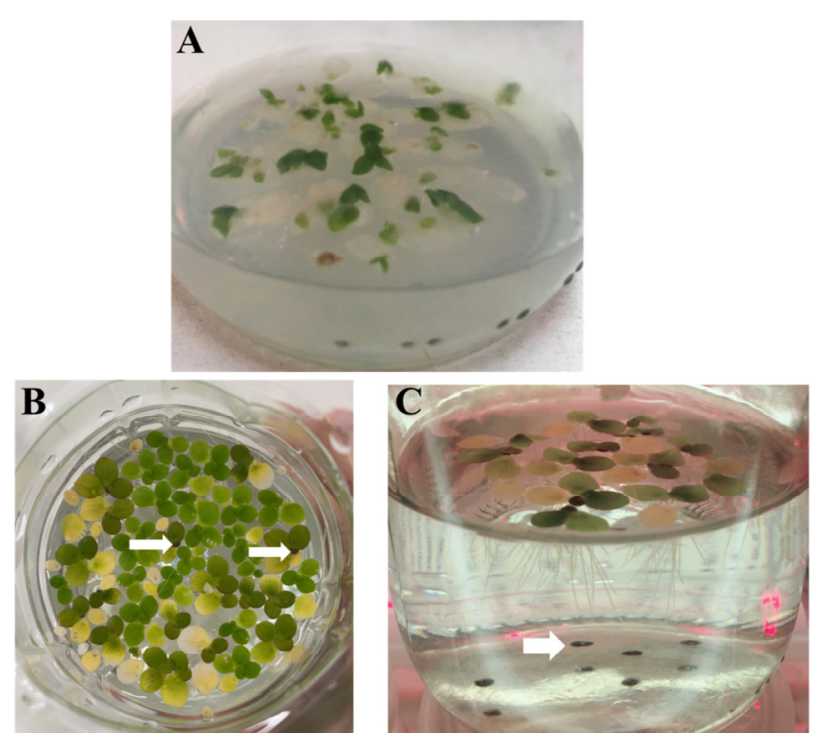

Fig. 2 S. polyrhiza culture and turion induction. (A) S. polyrhiza was cultured axenically for 14 days on MS medium. (B) Turions started to appear 7 days after induction via starvation and (C) completely sunk to the bottom of the vessel 45 days after induction. They were grown in $25 \pm 2^{\circ} \mathrm{C}$ with a $16 \mathrm{~h}$ light $/ 8 \mathrm{~h}$ dark photoperiod under $55 \mu \mathrm{mol} \mathrm{m} \mathrm{m}^{-2} \mathrm{~s}^{-1}$ fluorescent light bulbs 
PCR amplification of plasmid pB7WGY harboring $e Y F P$

The integration of target genes into the plasmid vectors was confirmed via PCR screening. The PCR products obtained from the amplification of plasmid $\mathrm{pB} 7 \mathrm{WGY}$ with gene-specific primers (eYFP-F and eYFP-R) showed positive bands of 298 base pairs (Fig. 3, lanes 4, 5 and 6). The plasmid pB7WGY vector harboring eYFP could then be used for recombinant protein expression, eYFP, through $A$. tumefaciensmediated turion transformation.

Transgenic turion and frond regeneration of S. polyrhiza

The frond regeneration of transgenic $S$. polyrhiza carrying the YYFP gene was tested on three media: MS1 for wild-type S. polyrhiza cultivation; MS2 with $250 \mathrm{mg} / \mathrm{L}$ ceftriaxone for Agrobacteria growth inhibition; and MS3 with 250 $\mathrm{mg} / \mathrm{L}$ ceftriaxone and $0.01 \mathrm{mM}$ glufosinate for transgenic S. polyrhiza selection. The proportions of transformants were $90 \%$ and $50 \%$, respectively, on the two types of media, and were significantly different between the selective media. Different co-culture conditions $16 \mathrm{~h}$ light $/ 8 \mathrm{~h}$ dark conditions, or dark co-cultivation of turions with Agrobacterium did not produce significant differences within the same selective medium (Table 1). The MS2 selective medium without glufosinate produced better frond regeneration under the $16 \mathrm{~h}$ light $/ 8 \mathrm{~h}$ dark conditions than MS3.

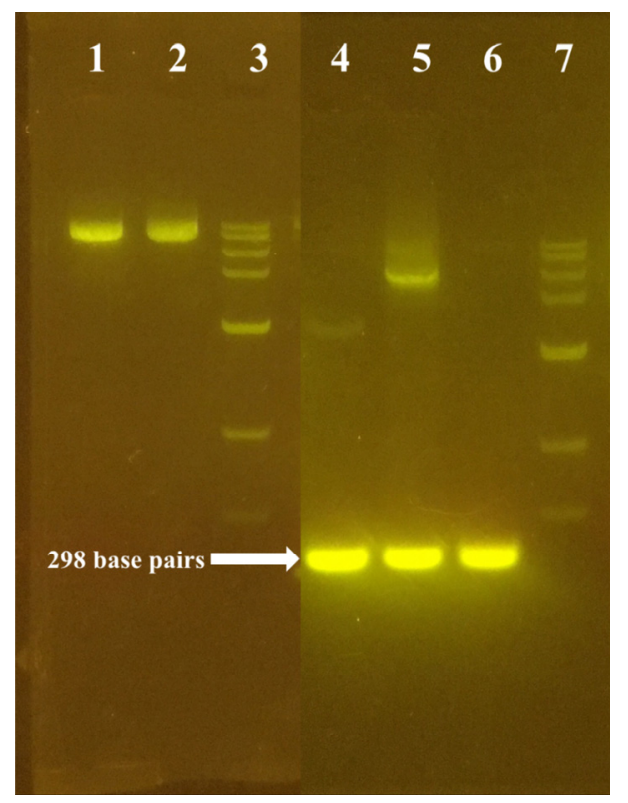

Fig. 3 Agarose gel electrophoresis of plasmid vectors and PCR products. Lanes 1 and 2, pB7WGY plasmids; lanes 3 and 6, 1.5 $\mathrm{kb}$ ladder; lanes 4-6, PCR amplification of eYFP gene-specific primers (eYFP-F and eYFP-R) showing the size of 298 base pairs, respectively
eYFP detection

The eYFP expression in transgenic duckweeds S. polyrhiza was detected after the regeneration of turions to fronds on selective media (MS2 and MS3), as demonstrated via the fluorescence of eYFP under the fluorescence microscope, compared with those from wild-type S. polyrhiza (Fig. 4A) and transgenic fronds of $S$. polyrhiza (positive control) (Fig. 4B). The autofluorescence of chlorophyll and fluorescence of eYFP were distinguished by the fluorescent colors, as the autofluorescence of chlorophyll was red when excited by green light (Fig. 4A-F). In addition, the fluorescence of eYFP was yellow when excited with green light, mixed with autofluorescence of chlorophyll (Fig. 4B-F). The results of frond regeneration on the selective media, MS2 and MS3, were clearly distinguishable from those on wild type (Fig. 4A-F). Transgenic S. polyrhiza were successfully transformed by employing the agroinfiltration method using turions of S. polyrhiza and observing yellow fluorescence. Co-cultivation with Agrobacterium was successful in transforming the eYFP protein in turions under $16 \mathrm{~h}$ light $/ 8 \mathrm{~h}$ dark conditions.
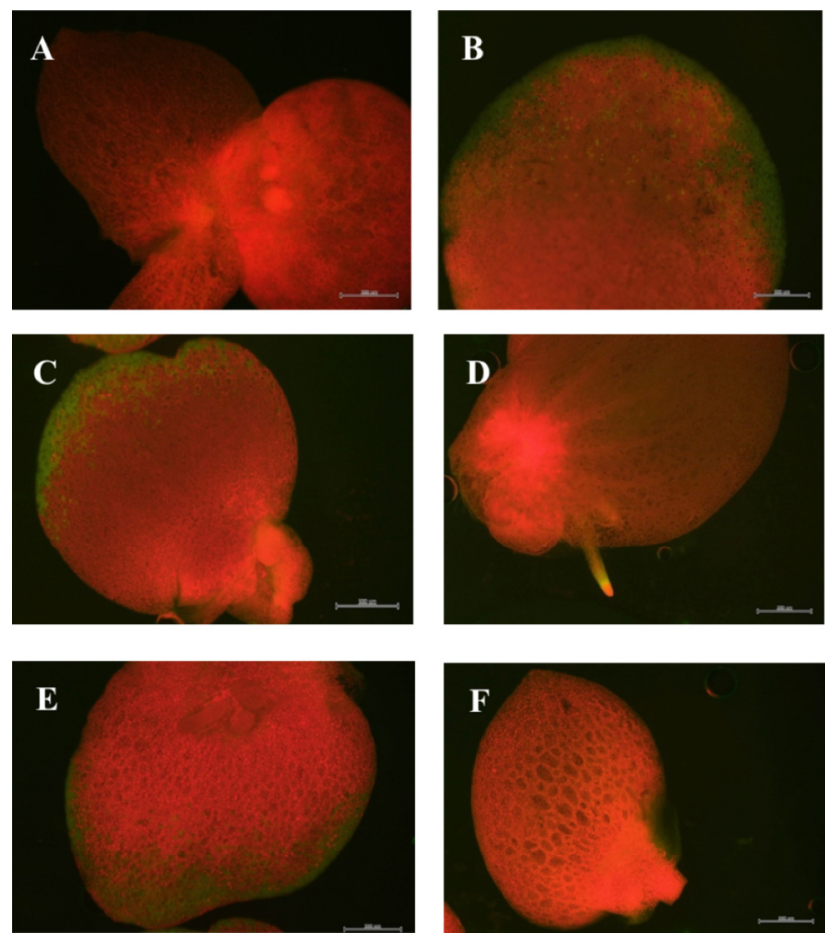

Fig. 4 eYFP fluorescence under the microscope (score bar = $500 \mu \mathrm{m})$ of frond regeneration on three types of MS media (MS1, MS2, and MS3) after cultivation for 14 days. (A) Wild type on MS medium. (B) Transgenic S. polyrhiza as a positive control. (C-D) Transgenic S. polyrhiza on MS2 and MS3 cocultivated with $A$. tumefaciens under $16 \mathrm{~h}$ light $/ 8 \mathrm{~h}$ dark conditions. (E-F) Transgenic $S$. polyrhiza on MS2 and MS3 co-cultivated with $A$. tumefaciens under dark conditions, respectively 
Table 1 The effect of selective media on frond regeneration and the transformation efficiency of S. polyrhiza turions. Co-cultivation represents

\begin{tabular}{|c|c|c|c|c|}
\hline $\begin{array}{l}\text { Selective } \\
\text { media }\end{array}$ & Co-cultivation & $\begin{array}{c}\text { Total number } \\
\text { of turions }\end{array}$ & $\begin{array}{c}\text { Number of } \\
\text { transformed cells }\end{array}$ & Transformation efficiency $(\%)$ \\
\hline \multirow{6}{*}{ MS2 } & & 10 & 10 & \multirow{3}{*}{$90.00 \pm 8.16^{\mathrm{A}}, \#, \# \#$} \\
\hline & $16 \mathrm{~h} \mathrm{light} / 8 \mathrm{~h}$ dark & 10 & 8 & \\
\hline & & 10 & 9 & \\
\hline & \multirow{3}{*}{ Dark } & 10 & 8 & \multirow{3}{*}{$83.33 \pm 4.71 *, * *$} \\
\hline & & 10 & 8 & \\
\hline & & 10 & 9 & \\
\hline \multirow{6}{*}{ MS3 } & & 10 & 6 & \multirow{3}{*}{$50.00 \pm 8.16^{\mathrm{A}}$} \\
\hline & $16 \mathrm{~h}$ light $/ 8 \mathrm{~h}$ dark & 10 & 5 & \\
\hline & & 10 & 4 & \\
\hline & \multirow{3}{*}{ Dark } & 10 & 5 & \multirow{3}{*}{$30.00 \pm 16.33$} \\
\hline & & 10 & 3 & \\
\hline & & 10 & 1 & \\
\hline
\end{tabular}

Each cultivation group consisted of 10 turions $(n=10)$. Percentage transformation efficiency is expressed as mean \pm standard deviation (SD) of three replicates. Uppercase letters (A) indicate no significant difference between the $16 \mathrm{~h}$ light $/ 8 \mathrm{~h}$ dark and dark cocultivation conditions on the same selective medium. The symbol \# indicates significant differences on the selective MS2 medium

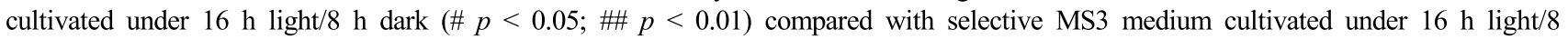
$\mathrm{h}$ dark and dark co-cultivation conditions, respectively. The symbol * indicates significant differences on the selective MS2 medium cultivated under dark $\left({ }^{*} p<0.05 ; *^{*} p<0.01\right)$ compared with selective MS3 medium cultivated under $16 \mathrm{~h}$ light/8 h dark and dark co-cultivation conditions, respectively.

\section{SDS-PAGE and western blot analysis}

The expression of the eYFP protein in transgenic $S$. polyrhiza was confirmed via western blot analysis using a monoclonal anti-rabbit GFP primary antibody. The SDS-PAGE profiles were generated for the total soluble proteins extracted from whole S. polyrhiza plants, including wild type and transformants. All protein samples were stained with Coomassie Brilliant Blue, and examples of western blot are presented in Fig. 5A and B. Non-specific bands were detected in the Coomassie Brilliant Blue-stained gel (Fig. 5A), and western blotting with a monoclonal anti-rabbit GFP primary antibody revealed an additional band at a molecular weight of about $28 \mathrm{kDa}$ when compared with the positive control transgenic fronds (Fig. 5B). The effects of the co-cultivation of turions with Agrobacterium under $16 \mathrm{~h}$ light $/ 8 \mathrm{~h}$ dark conditions and only dark conditions were compared. Co-cultivation under $16 \mathrm{~h} \mathrm{light} / 8 \mathrm{~h}$ dark conditions produced good eYFP protein expression on the selective MS2 medium and a pale band on selective MS3 medium during co-cultivation with Agrobacterium (Fig. 5B, lane 4 and 5), as well as good eYFP protein expression on the selective MS2 medium under dark conditions (Fig. 5B, lane 6) when compared with the positive control of transgenic duckweed. In contrast, no eYFP protein expression was observed on selective MS3 medium under dark conditions (Fig. 5B, lane 7), and no eYFP protein was detected in the wild type. This result indicated that the eYFP protein was successfully expressed and produced in the transgenic turions of S. polyrhiza via A. tumefaciens-mediated turion transformation under cocultivation under $16 \mathrm{~h}$ light $/ 8 \mathrm{~h}$ dark conditions to a greater extent than under dark conditions.

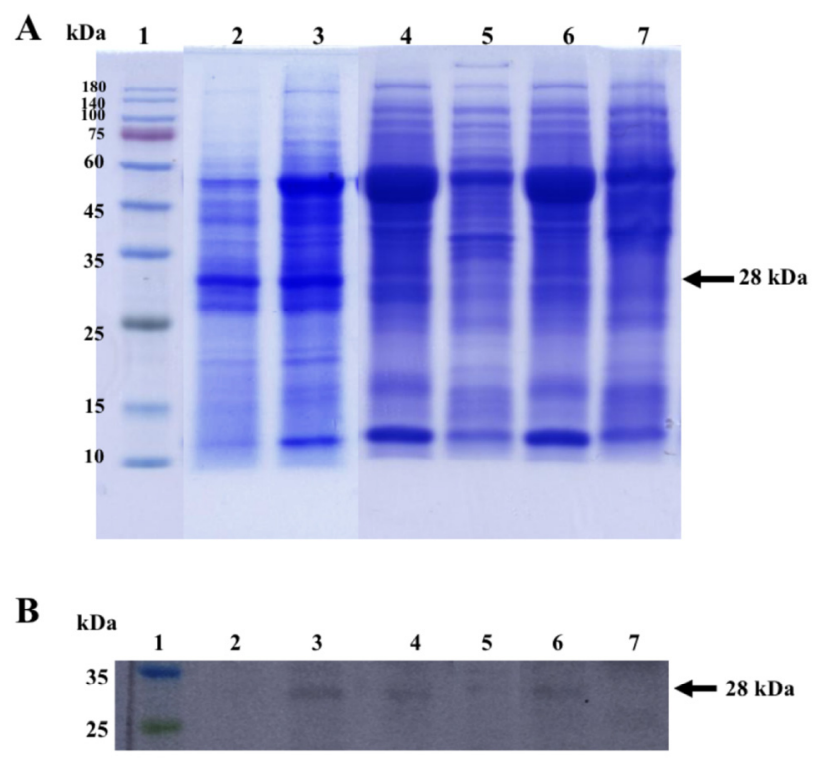

Fig. 5 SDS-PAGE and western blot analysis of the eYFP protein expression in turions. (A) SDS-PAGE. (B) Western blot: lane 1, prestaining protein marker; lane, 2, wild type; lane 3, transgenic duckweed as a positive control; lanes 4-5, transgenic duckweed on MS2 and MS3 co-cultivated with A. tumefaciens under 16 $\mathrm{h}$ light/ $8 \mathrm{~h}$ dark conditions; and lanes 6-7, transgenic duckweed on MS2 and MS3 co-cultivated with A. tumefaciens under dark conditions, respectively 


\section{Discussion}

We developed eYFP expressed in transgenic turions of $S$. polyrhiza to facilitate gene transformation. Some methods of transformation of Spirodela plants have been developed and refined to increase the transformation efficacy and stability of the expression of target genes (Vunsh et al. 2007). A. tumefaciens-mediated transformation is a highly effective method for gene transformation and protein production in duckweed plants (Firsov et al. 2015; Ko et al. 2011; Vunsh et al. 2007).

In this study, we report the expression of the eYFP protein in S. polyrhiza using A. tumefaciens-mediated turion transformation. The vector contains the $e Y F P$ gene, which encodes the eYFP protein, and has been extensively used as a reporter marker to visualize the gene expression in plants under the CaMV35S promoter with the bar gene as a selection marker to confer glufosinate resistance. Rapid transgenic-positive visual screening in the frond regeneration of turions in S. polyrhiza using this method provides a convenient method for visual screening under fluorescence microscope, and the protein expression was determined via western blotting. Different levels of red and yellow autofluorescence were observed in frond regeneration. The fronds regenerating from turions after 14 days demonstrated red autofluorescence due to the presence of chlorophyll, together with the part of the fronds emitting yellow autofluorescence from the transformants. The regenerated wild-type fronds were devoid of fluorescence. Western blotting confirmed that the target gene was actually expressed in the duckweed. The results confirmed the efficacy of the co-cultivation system used in this study. The eYFP protein exhibited good expression on the selective MS2 medium and produced a pale band on the MS3 medium under $16 \mathrm{~h}$ light $/ 8 \mathrm{~h}$ dark conditions but was not detected in wild-type plants. The performance of the co-cultivation of turions with Agrobacterium was compared under two different conditions: under a $16 \mathrm{~h}$ light $/ 8 \mathrm{~h}$ dark cycle and only in the dark. Light strongly promotes gene transfer from $A$. tumefaciens to plant cells (Zambre et al. 2003). However, Agrobacterium co-cultivation with duckweed plants resulted in a successful gene transformation under both conditions (Boehm et al. 2001; Vunsh et al. 2007; Yamamoto et al. 2001; Yang and Li et al. 2018; Yang et al. 2018).

In this study we confirmed that co-cultivation under $16 \mathrm{~h}$ light $/ 8 \mathrm{~h}$ dark conditions produced good eYFP expression, similar to that of positive control transgenic fronds, was detected using western blotting, and the efficiency of transformation was $90 \%$. The bar gene, which confers glufosinate resistance, is a selectable marker gene for generating transgenic dicotyledonous and monocotyledonous plants (Budhagatapalli et al. 2016; Penna et al. 2002; Zhang et al. 2018). Preliminary optimization studies of eGFP expression in turions were reported in our group, using a selective MS medium consisting of $0.01 \mathrm{mM}$ glufosinate, which efficiently screened out transformants with frond regeneration. The transformation frequency was detected as the frequency of bar and eGFP, and was up to 75\% (Jaiprasert 2018). However, the eYFP protein was not highly expressed on the selective medium that consisted of 0.01-mM glufosinate. These results indicated that the concentration of glufosinate should be lower than $0.01 \mathrm{mM}$ to regenerate fronds on selective medium. Otherwise, the presence of herbicide at high levels in the plant tissue culture would terminate wild-type cells and inhibit the growth of the transformants, leading to delays in plant regeneration (Boehm et al. 2001). The accumulation of herbicides in plant tissues exerted toxic and necrotic effects on plant cells by decreasing their growth and yield (Sikorski et al. 2019). High herbicide resistance might lead to the rapid natural development of plant populations (Rainbolt et al. 2004). Further studies are needed to investigate the reduction of herbicide concentration and stability of gene transformation in turions. Codon-optimized gene constructs could also be utilized to express proteins in the turions of $S$. polyrhiza for high protein expression levels.

\section{Conclusions}

We established a simple, novel transformation protocol for Spirodela polyrhiza via Agrobacterium tumefaciens-mediated turion transformation using the vacuum infiltration method. This system will provide a foundation for the genetic transformation of turions under co-cultivation with Agrobacterium tumefaciens in the $16 \mathrm{~h}$ light $/ 8 \mathrm{~h}$ dark conditions. These findings will pave the way for the use of duckweeds as tools in biotechnology and for therapeutic applications.

\section{Acknowledgments}

This work was financially supported by the Research Grant of Burapha University through National Research Council of Thailand [Grant no. 30/2558].

\section{References}

Appenroth KJ, Borisjuk N, Lam E (2013) Telling duckweed apart: 
Genotyping technologies for Lemnaceae. Chin J Appl Environ Biol 19:1-10

Áy Z, Mihály R, Cserháti M, Kótai É, Pauk J (2012) The Effect of High Concentrations of glufosinate ammonium on the Yield Components of Transgenic Spring Wheat (Triticum aestivum L.) Constitutively Expressing the bar Gene. Sci World J 2012:657945

Boehm R, Kruse C, Voeste D, Barth S, Schnabl H (2001) A transient transformation system for duckweed (Wolffia columbiana) using Agrobacterium-mediated gene transfer. J Appl Bot Food Qual 75(3):107-111

Budhagatapalli N, Schedel S, Gurushidze M, Pencs S, Hiekel S, Rutten T, Kusch S, Morbitzer R, Lahaye T, Panstruga R, Kumlehn J, Hensel G (2016) A simple test for the cleavage activity of customized endonucleases in plants. Plant Methods $12: 18$

Cantó-Pastor A, Mollá-Morales A, Ernst E, Dahl W, Zhai J, Yan Y, Meyers BC, Shanklin J, Martienssen R (2015) Efficient transformation and artificial miRNA gene silencing in Lemna minor. Plant Biol (Stuttg) 17 Supplement 1:59-65

Chhabra G, Chaudhary D, Sainger M, Jaiwal PK (2011) Genetic transformation of Indian isolate of Lemna minor mediated by Agrobacterium tumefaciens and recovery of transgenic plants. Physiol Mol Biol Plants 17(2):129-136

Christou P, Ford TL, Kofron M (1991) Production of transgenic rice (Oryza sativa L.) plants from agronomically important indica and Japonica varieties via electric discharge particle acceleration of exogenous DNA into immature zygotic embryos. Nat Biotechnol 9(10):957-962

Dröge W, Broer I, Pühler A (1992) Transgenic plants containing the phosphinothricin-N-acetyltransferase gene metabolize the herbicide L-phosphinothricin (glufosinate) differently from untransformed plants. Planta 187(1):142-151

Firsov A, Tarasenko I, Mitiouchkina T, Ismailova N, Shaloiko L, Vainstein A, Dolgov S (2015) High-yield expression of M2e peptide of avian influenza virus H5N1 in transgenic duckweed plants. Mol Biotechnol 57(7):653-661

Gordon-Kamm WJ, Spencer TM, Mangano ML, Adams TR, Daines RJ, Start WG, O’Brien JV, Chambers SA, Adams WR Jr, Willetts NG, Rice TB, Mackey CJ, Krueger RW, Kausch AP, Lemaux PG (1990) Transformation of maize cells and regeneration of fertile transgenic plants. Plant Cell 2(7): 603-618

Hillman WS (1961) The Lemnaceae, or duckweeds: A review of the descriptive and experimental literature. Bot Rev 27(2): 221-287

Jaiprasert A (2018) Development of duckweed transformation technique for biological application $\mathrm{PhD}$ thesis BURAPHA UNIVERSITY

Janakiraman V, Steinau M, McCoy SB, Trick HN (2002) Recent advances in wheat transformation. In Vitro Cell Dev Biol Plant 38(5):404-414

Jefferson RA, Kavanagh TA, Bevan MW (1987) GUS fusion: $\beta$ glucuronidase as a sensitive and versatile gene fusion marker in higher plants. EMBO J 6(13):3901-3907
Jusuk I, Vietz C, Raab M, Dammeyer T, Tinnefeld P (2015) Super-Resolution Imaging Conditions for enhanced yellow fluorescent Protein (eYFP) Demonstrated on DNA Origami Nanorulers. Sci Rep 5:14075

Karimi M, Inzé D, Depicker A (2002) Gateway vectors for Agrobacterium-mediated plant transformation. Trends Plant Sci 7(5):193-195

Ko SM, Sun HJ, Oh MJ, Song IJ, Kim MJ, Sin HS, Goh CH, Kim YW, Lim PO, Lee HY, Kim SW (2011) Expression of the protective antigen for PEDV in transgenic duckweed, Lemna minor. Hortic Environ Biotechnol 52(5):511

Krajnčič B, Devidé Z (1979) Flower development in Spirodela polyrrhiza (Lemnaceae). Plant Syst Evol 132(4):305-312

Krajnčič B, Slekovec-Golob M (1991) Synergistic effect of GA3 and EDDHA on the promotion of flowering in the photoperiodically neutral plant Spirodela polyrrhiza (L.) Schleiden. J Plant Physiol 139(2):240-242

Landolt E (1986) The family of Lemnaceae - A monographic study. Biosystematic Investigations in the family of duckweeds (Lemnaceae). Veröffentlichungen des Geobotanischen Institutes der ETH Rübel, Zurich, Switzerland 1

Landolt E, Kandcler R (1987) The Family of Lemnaceae - A Monographic Study. Biosystematic. Investigations in the Family of Duckweeds (Lemnaceae) Ver6ff geobot Inst ETH, Stiflung Riibel, Z rich 2

Lemon GD, Posluszny U (2000) Comparative shoot development and evolution in the Lemnaceae. Int J Plant Sci 161(5): 733-748

Lemon GD, Posluszny U, Husband BC (2001) Potential and realized rates of vegetative reproduction in Spirodela polyrhiza, Lemna minor, and Wolffia borealis. Aquat Bot 70(1):79-87

Mejbel HS, Simons AM (2018) Aberrant clones: Birth order generates life history diversity in Greater duckweed, Spirodela polyrhiza. Ecol Evol 8(4):2021-2031

Oláh V, Tóth G, SzöIlôsi E, Kiss T (2008) Comparative study on sensitivity of different physiological properties of Spirodela polyrrhiza (L.) Schleiden to Cr(VI) treatments. Acta Biol Szeged 52:181-182

Ormö M, Cubitt AB, Kallio K, Gross LA, Tsien RY, Remington SJ (1996) Crystal structure of the Aequorea victoria green fluorescent protein. Science 273(5280):1392-1395

Penna S, Sági L, Swennen R (2002) Positive selectable marker genes for routine plant transformation. In Vitro Cell Dev Biol Plant 38(2): 125-128

Rainbolt CR, Thill DC, Yenish JP, Ball DA (2004) Herbicideresistant grass weed development in imidazolinone-resistant wheat: Weed biology and herbicide rotation. Weed Technol 18(3):860-868

Sambrook J, Fritsch EF, Maniatis T (1989) Molecular cloning. A laboratory manual (2nd ed) Cold Spring Harbor, NY: Cold Spring Harbor Laboratory Press

Sikorski Ł, Baciak M, Bęś A, Adomas B (2019) The effects of glyphosate-based herbicide formulations on Lemna minor, a non-target species. Aquat Toxicol 209:70-80 
Tan S, Evans R, Singh B (2006) Herbicidal inhibitors of amino acid biosynthesis and herbicide-tolerant crops. Amino Acids 30(2): 195-204

Tang J, Zhang F, Cui W, Ma J (2014) Genetic structure of duckweed population of Spirodela, Landoltia and Lemna from Lake Tai, China. Planta 239(6):1299-1307

Thu PTL, Huong PT, Tien VV, Ham LH, Khanh TD (2015) Regeneration and transformation of gene encoding the hemagglutinin antigen of the H5N1 virus in frond of duckweed (Spirodela polyrhiza L.). J Agric Res 3 (1)

Tsien RY (1998) The green fluorescent protein. Annu Rev Biochem 67:509-544

Vasil V, Castillo AM, Fromm ME, Vasil IK (1992) Herbicide resistant fertile transgenic wheat plants obtained by microprojectile bombardment of regenerable embryogenic callus. Nat Biotechnol 10(6):667-674

Vunsh R, Li J, Hanania U, Edelman M, Flaishman M, Perl A, Wisniewski JP, Freyssinet G (2007) High expression of transgene protein in Spirodela. Plant Cell Rep 26(9):1511-1519

Wang W, Haberer G, Gundlach H, Gläßer C, Nussbaumer T, Luo MC, Lomsadze A, Borodovsky M, Kerstetter RA, Shanklin J, Byrant DW, Mockler TC, Appenroth KJ, Grimwood J, Jenkins J, Chow J, Choi C, Adam C, Cao XH, Fuchs J, Schubert I, Rokhsar D, Schmutz J, Michael TP, Mayer KF, Messing J (2014) The Spirodela polyrhiza genome reveals insights into its neotenous reduction fast growth and aquatic lifestyle. Nat Commun 5:3311

Wang W, Messing J (2012) Analysis of ADP-glucose pyrophosphorylase expression during turion formation induced by abscisic acid in Spirodela polyrhiza (greater duckweed). BMC Plant Biol 12(5):5
Wang W, Yang C, Tang X, Zhu Q, Pan K, Cai D, Hu Q, Ma D (2015) Carbon and energy fixation of great duckweed Spirodela polyrhiza growing in swine wastewater. Environ Sci Pollut Res Int 22(20):15804-15811

Wang Y (2016) Callus induction and frond regeneration in Spirodela polyrhiza. Czech J Genet Plant Breed 52 (3): 114-119

Yamamoto YT, Rajbhandari N, Lin X, Bergmann BA, Nishimura Y AM, Stomp A (2001) Genetic transformation of duckweed Lemna gibba and Lemna minor. In Vitro Cell Dev Biol Plant 37(3):349-353

Yang GL, Fang Y, Xu YL, Tan L, Li Q, Liu Y, Lai F, Jin YL, Du AP, He KZ, Ma XR, Zhao H (2018) Frond transformation system mediated by Agrobacterium tumefaciens for Lemna minor. Plant Mol Biol 98(4-5):319-331

Yang GL, Feng D, Liu YT, Lv SM, Zheng MM, Tan AJ (2021) Research progress of a potential bioreactor: Duckweed. Biomolecules 11(1)

Yang J, Li G, Hu S, Bishopp A, Heenatigala PPM, Kumar S, Duan P, Yao L, Hou H (2018) A protocol for efficient callus induction and stable transformation of Spirodela polyrhiza (L.) Schleiden using Agrobacterium tumefaciens. Aquat Bot 151:80-86

Zambre M, Terryn N, De Clercq J, De Buck S, Dillen W, Van Montagu M, Van Der Straeten D, Angenon G (2003) Light strongly promotes gene transfer from Agrobacterium tumefaciens to plant cells. Planta 216(4):580-586

Zhang J, Yin K, Sun J, Gao J, Du Q, Li H, Qiu JL (2018) Direct and tunable modulation of protein levels in rice and wheat with a synthetic small molecule. Plant Biotechnol J 16(2):472-481 